\title{
La ligereza como nueva forma de vida: entrevista con Gilles Lipovetsky para un análisis del presente
}

EDWARD SALAZAR*

Entrevista académica al sociólogo Guilles Lipovetsky centrada en el tema de sociedad contemporánea y consumo.

Recibida: 27 de septiembre del 2019 - Evaluada: 12 de mayo del 2020 - Aceptada: 18 de mayo del 2020

Citar como: Salazar, E. (2020). La ligereza como nueva forma de vida: entrevista con Gilles Lipovetsky para un análisis del presente. Hallazgos, 17(34), 305-320. DoI: https://doi.org/10.15332/2422409X.533

\footnotetext{
Magíster en Estudios Culturales. Docente investigador de la Universidad Santo Tomás, Sede Principal, Colombia.

Correo electrónico: efsalazarc@gmail.com

ORCID: 0000-0001-5456-9962
} 


\section{Resumen}

En esta entrevista el sociólogo francés Gilles Lipovetsky, mundialmente reconocido por sus trabajos sobre la moda, el consumo y las sociedades hipermodernas, analiza algunos de los aspectos transversales de su obra, especialmente sobre la última de sus contribuciones analíticas: la sociedad de la ligereza. Introduzco esta entrevista con una lectura crítica a las teorías de Lipovetsky para contextualizar y problematizar sus postulados, con miras a recoger las contribuciones del autor que permitan un análisis contextualizado del presente.

Palabras clave: consumo, Gilles Lipovetsky, moda.

\section{Lightness as a new way of life: interview with Gilles Lipovetsky for an analysis of the present}

\section{Abstract}

In this interview, French sociologist Gilles Lipovetsky, world-renowned for his work on fashion, consumption and hypermodern societies, analyzes some of the cross-cutting aspects of his work, especially on the last of his analytical contributions: the society of lightness. I introduce this interview with a critical reading of Lipovetsky's theories to contextualize and problematize his postulates, with a view to collecting the author's contributions that allow a contextualized analysis of the present.

Keywords: consumption, Gilles Lipovetsky, fashion.

\section{A leveza como nova forma de vida: entrevista com Gilles Lipovetsky para uma análise do presente \\ Resumo}

Nesta entrevista, o sociólogo francês Gilles Lipovetsky, reconhecido no mundo inteiro por seus trabalhos sobre a moda, o consumo e as sociedades hipermodernas, analisa alguns dos aspectos transversais de sua obra, especialmente sobre a última de suas contribuições analíticas: a sociedade da leveza. Introduzo esta entrevista com uma leitura crítica das teorias de Lipovetsky para contextualizar e problematizar seus princípios com vistas a coletar as contribuições do autor que permitam uma análise contextualizada do presente.

Palavras-chave: consumo, Gilles Lipovetsky, moda. 
La obra del francés Gilles Lipovetksy (n. 1940), a medio camino entre el ensayo de divulgación científica y la teoría social densa, ha gozado de una amplia recepción en el campo de los estudios de la contemporaneidad, en lo que el autor ha llamado el análisis de la hipermodernidad. Su manera de indagación y escritura parten del método genealógico nietzscheano y foucaultiano en cuanto a la necesidad de desentrañar analíticamente el desarrollo histórico de las estructuras sociales y de poder, desde lo que el autor denominó como "análisis de lo paradójico” (Corral, 2007, p. 41). Lipovetksy parte de la identificación de situaciones socioculturales de contraste, para abordar las tensiones y contradicciones que se tejen en fenómenos sociales que se oponen, pero que de ninguna manera se excluyen. Por ejemplo, las tensiones entre la libertad que trae el consumo como expresión de la subjetividad, al tiempo que la opresión que significa la construcción de la identidad basada en las mercancías.

Las estrategias y analíticas de Lipovetksy han sido particularmente atractivas debido a los problemas contemporáneos en los que el autor centra su atención, que son en últimas la nueva democracia desde el consumo y su papel en la expresión de la individualidad. Lipovetksy parte del análisis de las nuevas estructuras políticas, mucho menos rígidas frente a las de la modernidad de las instituciones sólidas, que analiza en profundidad en La era del vacío (1986), su primera obra de amplia difusión. El autor recalca el paso de la modernidad a la posmodernidad, pero sobre todo a lo que llama la hipermodernidad, que se trata de un escenario en el que la individualidad, el consumo y la autoexpresión constituyen los pilares de las relaciones sociales, en las que el yoautoreferencial opaca el sentido del deber con arreglo a las grandes pautas sociales características de la modernidad. La sociedad hipermoderna es la sociedad del hiperconsumo, de la inflación del yo. A partir de entonces, Lipovetsky se ha centrado en su análisis, haciendo hincapié en cada uno de sus libros en un fenómeno particular (pero todos profundamente relacionados), tales como la moda, el lujo, la feminidad, la sociedad de la ligereza, la política, entre otros temas.

Críticos de la obra del autor reconocen los aportes discursivos que trae el hablar de la hipermodernidad debido al triunfo del capitalismo del consumo y de la subjetividad. Sin embargo, también se aproximan críticamente a las teorizaciones generalistas del autor y su agenda intelectual:

La hipermodernidad es un término que genera más confusión que claridad, aunque seguramente haya nacido para ello, para remover las aguas comerciales primero francesas y luego mundiales, porque cuanto más se enturbian esas aguas mejor pescan los especialistas en las industrias culturales y en la fabricación de best-sellers sociológicos de baja densidad científica. (Alonso y Fernández, 2010, p. 332) 
Esta sugerente afirmación puede verse patente en Colombia. Lipovetksy ha estado en Colombia en diferentes ocasiones: en 2002, en Bogotá, Medellín y Cali; en 2003, en Medellín, Manizales y Bogotá; en 2010, en la Universidad de Cartagena y en la Feria Internacional del Libro de Bogotá (Filbo); en 2015, en Bogotá en la Biblioteca Luis Ángel Arango; en 2016, invitado por la Personería de Bogotá y la Universidad Externado de Colombia; en 2017, en la Universidad Pontificia Bolivariana de Medellín; en 2019, nuevamente en la Filbo y en el XI Encuentro Internacional de Periodismo (de la Universidad Externado de Colombia).

Sus conferencias y charlas, además de presentar sus novedades editoriales, han girado principalmente en torno al desarrollo de la hipermodernidad, sin resultar sorprendente, puesto que este es uno de los temas que le han otorgado la notoriedad académica y mediática de la que goza el autor. Al tiempo, en Colombia ha sido principalmente indagado sobre los fenómenos del consumo, la subjetividad, la feminidad y la moda, que son desarrollos que Lipovetsky hace dentro de su análisis paradójico de la hipermodernidad. Aunque su obra ha pasado por distintas etapas, que Corral (2007) periodiza en tres, a saber: etapa autocrítica marxista (1965-1983), etapa posmoderna (1983-1991), etapa hipermoderna (1992-), el núcleo de su pensamiento ha sido constante: el cambio paradigmático de la modernidad capitalista y occidental, de las instituciones estables y duraderas, a la sociedad hipermoderna que privilegia lo efímero. La modernidad capitalista había sido ampliamente estudiada por Weber frente a la familia, la religión y el Estado, mientras que Bauman analizó la sociedad líquida en la que la caducidad y las relaciones personales fugaces son valores fundamentales. Es decir, Lipovetsky ha mantenido el centro de su análisis en dicho cambio de valores, bien desde una mirada estructural marxista, o en el fenómeno de la moda como epítome de la hipermodernidad, caracterizadas por desarrollar estructuras políticas de libertad democrática capitalistas, de placer inmediato y por la hegemonía de la individualidad como nuevas epistemes favorecidas por el consumo masivo.

Sin duda los aportes lipovetskianos gozan de una vigencia indiscutible tanto por la actualidad de sus ideas para el análisis del presente como por su lugar visible en la academia y en los medios de comunicación masivos. La sociedad de los consumidores está en su pleno apogeo, especialmente en contextos como el colombiano, en el que las clases medias se encuentran en un proceso de asentamiento y paulatina consolidación, a través del acceso a nuevos estilos y formas de vida por medio de la democratización del consumo. La moda y el acceso a ella es sin duda uno de los mayores escenarios en los que la sociedad hipermoderna expresa sus valores de individualidad, apariencia democrática y favorecimiento de la inmediatez, tal como 
lo desarrolló en detalle Lipovetsky en El imperio de lo efímero (1987) y El lujo eterno (2003, coescrito con Roux), principalmente. Su último libro, De la ligereza (2016), pone en funcionamiento el aparataje analítico y ensayístico que ha desarrollado en su carrera, y analiza el fenómeno de relajación en la profundidad de los discursos y prácticas en terrenos como la política, el consumo, la moda y la arquitectura, la familia, los medios de comunicación, entre otros. Esta es, en últimas, una mirada que continúa con sus análisis de la frivolidad, por lo que la ligereza vuelve y desarrolla los asuntos ya conocidos en el resto de su obra.

Lipovetsky señala que la sociedad contemporánea ha favorecido la construcción de un estilo de vida orientado a la búsqueda sencilla de la felicidad, y en consecuencia el alejamiento de los sufrimientos cotidianos. Es decir, aligerar el peso de la existencia. Esta premisa ha desencadenado tanto en la búsqueda del placer inmediato, el favorecimiento de experiencias livianas en todo sentido, como por ejemplo la práctica superficial por parte de muchas personas de espiritualidades y corporalidades como el yoga, que se convierten en consumos superfluos del bienestar. Incluso, señala, la ligereza se percibe en el diseño de muebles casi aéreos, moda dominada por los llamados "básicos", o arquitecturas sinuosas, casi virtuales, dominadas por el vidrio. La ligereza es una forma de adornar la vida y despojarla de gravedad. Aunque la ligereza frivoliza el mundo, al tiempo libera a los individuos de cargas imposibles de llevar, como el corresponder a ideales tradicionales de familia, religiosidad, sexualidad, etc. Como lo ha hecho a lo largo de su obra, Lipovetsky juega con las paradojas, y en la ligereza señala que la alegría, efímera, podría convertirse también en una opresión.

La ligereza es evidente en muchas esferas de la vida cotidiana en múltiples contextos mundiales, y de ello podría ser indicador irrefutable una gran parte de lo que ocurre en una red social como Instagram. Allí está a la orden del día la promoción del bienestar emocional superficial, la espiritualidad ligera en poses de yoga, la redistribución de la riqueza reducida a la caridad efímera, o la lucha política del feminismo reducida a una estetización frívola y ligera. También la aligeración de los debates sobre desigualdad estructural por medio de la diseminación ingenua de eslóganes de consumo como "que el privilegio no nuble la empatía", como se vio en Colombia, que hace atractiva y consumible una postura en apariencia política, pero que despoja de densidad la discusión frente a las clases sociales, la inequidad y la naturalización como "privilegio", lo que constitucionalmente es un derecho. Se trata de la estetización superflua de ideas políticas, en las que el debate pierde su lugar por medio de abanderadas y abanderados de la felicidad y la conciencia política ligera. 
Sin embargo, y más allá de lo ligero, a la sociedad de los productores consumidores masivos le han surgido nuevas preguntas que contribuyen al avance de lo que puede llamarse la sociología del presente, más allá de la mirada sobre la hipermodernidad y con preguntas de densidad política. Por ejemplo, en el campo de la moda y de los espectáculos son visibles los debates y activismos que reclaman responsabilidades ambientales, aquellos que denuncian las condiciones de explotación de las maquilas, los activismos frente a las corporalidades diversas o los movimientos de mujeres y feminismos frente a fenómenos masivos como el Me Too, más allá de lo planteado por Lipovetsky en su libro La tercera mujer (1997). Aquí es necesario detenerse y señalar, por un lado, las críticas feministas hechas a La tercera mujer de Lipovetsky, y en general a la mirada del autor frente a las mujeres y lo femenino, como la de Luisa Posada (2019). Para ella, la visión de las mujeres de Lipovetsky es sesgada y reduccionista, entre otras razones porque está lejos del reconocimiento del problema estructural del patriarcado y del reconocimiento de las opresiones aún vividas por las mujeres, lo que reduce su lectura a un asunto de liberación desde el consumo. Por ende, para la autora, su obra desdeña del feminismo, limita la comprensión de las mujeres, y en consecuencia estaría lejos de la perspectiva feminista. Las respuestas Lipovetsky en esta entrevista permiten ver estas tensiones.

Por otro lado, frente al movimiento Me Too, Lipovetsky aborda el tema como pensador internacional a los fenómenos masivos más notorios, como lo ha hecho a lo largo de su obra crítica, no desde una lectura en clave feminista. No profundiza (en parte por la naturaleza misma de la entrevista) en las dimensiones políticas y genealógicas estructurales de dicho movimiento. No lo deslegitima, pero sí cuestiona la legalidad y eticidad que pudiera estar comprometida en una acusación infundada. Por el contrario, movimientos de mujeres, de manera individual u organizada, han defendido la voz y el testimonio de las mujeres ante una sociedad patriarcal cómplice con las violencias de género. En esta perspectiva, la credibilidad en la voz de las mujeres es la base de las denuncias, así como se defiende la necesidad de la sanción social ante los problemas de acceso a la justicia.

En Colombia, el debate al respecto ha sido amplio en redes sociales y medios de comunicación masivos, con voces de mujeres feministas que reconocen que no toda visibilización de las experiencias de las mujeres son un Me Too. La escritora feminista Gloria Susana Esquivel ha sido vocal en diferentes medios y espacios de estos mecanismos legítimos de defensa y empatía ante unas historias que cargan heridas y ante un sistema de justicia patriarcal. La activista y pensadora feminista Lorena Aristizábal reconoce las complejidades que tiene la denuncia pública en las redes sociales y 
su inmediatez irreflexiva en su tono espectacular que reduce el debate, la necesidad de pensar alternativas feministas a la simple exposición mediática, al tiempo que señala que la credibilidad en la voz de las mujeres va de la mano de la necesidad de replantear las nociones de debilidad y revictimización de las mujeres. Sin embargo, señala, en estos procesos hay que comprender tanto los momentos y niveles para radicalizar la respuesta, como aquellos posteriores para promover la profundización en los problemas.

También se han sumado voces que rechazan algunas de las acusaciones por ser infundadas, o por poner bajo sospecha cualquier acto erótico que puede ser entendido como consensuado. La escritora feminista Carolina Sanín señaló a este respecto: "De ninguna manera creo que la ola [del Me Too] deba morigerarse, ni acogerse a un término medio. Lo que creo es, por el contrario, que el feminismo debe redefinir todos los términos y exigir la absoluta equidad" (Sanín, 2018, párr. 12). Es decir, no se cuestiona el Me Too, pero sí se complejiza el procedimiento o el lugar de enunciación que podría esencializar a las mujeres como personas aminoradas y desprovistas de autonomía. El debate es complejo y no puede ser abordado acá, y aunque Lipovetsky señala en la entrevista algunas de sus paradojas, el debate debe estar en cabeza de las mujeres feministas. Acá presento solo algunos de los puntos recogidos en el debate feminista, al tiempo que señalo de la mano de Lipovetsky el fenómeno de aligeramiento que producen las redes sociales junto con su potencia como lugar de enunciación.

A pesar de que Lipovetsky es uno de los autores más reconocidos y citados, tanto en la academia científica como en esferas mediáticas del conocimiento, pues sus ideas permiten marcos comprensivos generales a la sociedad contemporánea, es necesario siempre un ejercicio de contextualización y problematización, por ejemplo, en los casos latinoamericanos. Así, frente a De la ligereza cabe mencionar fenómenos que se escapan a esta visión de un mundo desprovisto de densidad: los movimientos sociales en América Latina, que desde políticas ambientales cuestionan la industria de la moda, los movimientos feministas y queer, que leen el cuerpo y la acción colectiva por fuera del proyecto de individualización en el que tanto insiste Lipovetsky.

La generalización no es un problema solo de este autor, sino también de las teorías macro de lo social, especialmente aquellas de corte anglo-eurocéntricas, que proveen al tiempo un marco válido para la interpretación de un fenómeno, a la vez que limitan su comprensión: parten de un sujeto universal que no es vivido en todos los contextos de la misma manera. Las teorías decoloniales y los pensamientos de América Latina hacen contrapesos a estas epistemologías únicas. Sin embargo, la 
obra de Lipovetsky ha sido fundamental para los estudios de la juventud, la hipermodernidad y la moda, por lo que sus aproximaciones continúan siendo, para muchas y muchos, marcos de interpretación vigentes y pertinentes.

De abordar esas pertinencias va esta entrevista. Contextualizarlas, problematizarlas o cuestionarlas es una opción que queda al lector y la lectora.

E. SAlAZAR: De La tercera mujer a De la ligereza se puede establecer una continuidad en su idea de "desustanciación de lo político", es decir de frivolización de la política. Sin embargo, la moda, la feminidad y la política han mostrado sus lazos estrechos...

G. LIPOVETKSY: La pregunta sobre lo político me parece el vínculo más nuevo, más reciente, que ha tenido la moda. Pero se trata de una política en el sentido noble de la palabra, es decir, una política civilizacional, y se establece un vínculo natural o evidente entre la moda y la exigencia ecológica. Es nuevo porque desde que existe la moda en Occidente, es decir, desde finales de la Edad Media, la moda traduce civilidades, sensibilidades, estéticas culturales, pero sin ambición política. La moda es el arquetipo de la frivolidad. Ha sido históricamente un sector de la vida social que invita al juego de las apariencias, sin tener necesariamente ambiciones de transformación profunda.

A pesar de todo, hay que decir que a partir del siglo xx puede rastrearse un esbozo de vínculo entre moda y política, con lo que se produce como una liberación femenina entendida como proceso de transformación. Chanel, entre otras (aunque Chanel no fue nada política), decía querer liberar a las mujeres de un sistema opresivo para el cuerpo, de manera que su apuesta de diseño se trata de política en cierto sentido. Por medio de la ropa, de vestidos ligeros, una mujer se libera, le es posible la movilidad cotidiana, y por ende puede ir a trabajar. Todo ello demuestra una voluntad de emancipación, pero una emancipación que está todavía en el campo de la elegancia.

Por otro lado, hoy en día hay, podemos decir que se ha desarrollado un nuevo escalón por medio del cual estamos hablando de la moda y la política desde un terreno, digamos, mucho más serio. La moda, como las demás industrias de consumo, ha dado cuenta de que su actividad tal como existe es nociva para el equilibrio planetario, así que no puede negar su contribución dañina, por ejemplo, al calentamiento global. Así pues, existe una voluntad de inventar una moda de un modo que sea responsable. Si unimos las palabras, moda y responsabilidad, a simple vista podríamos decir que se trata casi de un oxímoron: cómo así que la moda es responsable. Pero, hoy en día, podemos decir que sí podemos tener estas palabras juntas, pues se está hablando colectivamente de moda responsable y de lujo responsable. 
Esto significa que los materiales que se usan para la confección de las prendas están propendiendo hacia una revolución completa en pos de materiales sostenibles y responsables ambientalmente. De todas maneras, este marco de acción acelera la enorme ansiedad que se vive en nuestra época, porque el fenómeno frívolo por excelencia que es la moda está dándose cuenta de la tendencia que tiene en cuenta el equilibrio ambiental del planeta. Esto crea una relación problemática y ambigua que debe ser atendida. Desde mi perspectiva, esto es lo que hay quizás más nuevo sobre el tema de la moda, pues no tiene que ver esencialmente con el estilo, con lo efímero, sino que se trata de algo mucho más profundo.

En cuanto al feminismo, hay que hacer una distinción entre lo que sucede en las universidades estadounidenses donde existen múltiples escuelas en estudios de lo femenino y en estudios de género como especialidades académicas. Por otro lado, hay que considerar el movimiento social, en donde no es seguro y no es certero que ambos lados sean necesariamente la misma cosa. Tengo que decir que a mílo que me interesa más es el movimiento social. Los estudios de género son un asunto mucho más sofisticado, y tiende a haber textos extremistas. Recordemos textos célebres en los que se dice prácticamente que cualquier relación sexual es una violación, como si entre los hombres y las mujeres hubiera una guerra. Las considero posiciones extremas, y no estoy seguro de que la mayoría de las mujeres busquen exactamente esas polémicas. En cambio, en cuanto al movimiento social, me parece interesante para el análisis la problemática que desata lo que podría llamarse feminismo pop.

Quizás el fenómeno más marcante es el del Me Too. Es sumamente importante porque se trata de muchas mujeres que estuvieron allí, no solo académicas, sentando una voz de denuncia. Respecto de este fenómeno, considero que existe una faceta positiva y otra que lo es menos. La parte positiva es que a pesar de todo las mujeres siguen luchando para rechazar las opresiones impuestas por los hombres. El movimiento Me Too es una prolongación del movimiento de emancipación femenina, una emancipación que tiene que ver con la vida diaria. No tiene tanto que ver con la ley. En todas partes, cuando hay acoso, es decir, donde las mujeres se sienten incómodas, se sienten agredidas, el tomar la palabra es para las mujeres una de las formas en las que hacen retroceder esos comportamientos que para los hombres han sido naturalizados, y que por supuesto y sin lugar a dudas para para las mujeres no lo son. Son violentos.

De tal forma que me parece que el hecho de que haya liberación de la palabra puede ser una manera fundamental que haga que los hombres jóvenes reflexionen y se hagan más preguntas frente a sus acciones y creencias. Hará también que las 
empresas sean más cuidadosas, precisamente estableciendo reglas que en teoría han existido, pero que deben aplicarse seriamente, por ejemplo, frente al acoso sexual. Las mujeres, particularmente en su trabajo, quieren ser tratadas como seres que trabajan y no como objetos sexuales.

Así que el fondo de estas acciones es totalmente legítimo, y forma parte de la profundización de la dinámica de individualización. En otras palabras, del proceso de conquista amplia y progresiva de la autonomía por parte de las mujeres. Es decir: cómo ser autónoma si el jefe jerárquico está encima mío.

Como primera medida, hay que preguntarse si en el movimiento Me Too no cabrían algunas reflexiones críticas. Yo creo que sí. Lo primero que está puesto en el fenómeno es que el público empieza a reemplazar a la justicia. A las redes sociales se lanzan condenas, críticas y acusaciones, sin la posibilidad de respuesta. En alguno $\mathrm{u}$ otro momento la justicia tiene que actuar, porque de lo contrario lo que reina es lo arbitrario. Diría que con relación al feminismo eso no sería lo más preocupante. Lo que no es bueno es que, a pesar de todo, sin quererlo, este fenómeno repite el arquetipo de la mujer víctima. Con el movimiento Me Too todas las mujeres se quejan de estar agredidas, de la maldad de los hombres, y por ende se reproduce el relato de las mujeres como víctimas, desprovistas de autonomía.

Como segunda medida, las mujeres han sido víctimas en experiencias que efectivamente pasaron, pero tampoco son una abominación. Una cosa es la existencia de actos de violencia física o de violencia sexual por parte, por ejemplo, de un jefe o de un superior jerárquico. Pero pensemos en una fiesta, en una reunión. Las personas han bebido más de la cuenta y existe alguna broma pasada del tono. Es compresible que a una mujer no le guste el comentario, pero no asumo el considerar un comentario seductor fuera de ese marco. Una mujer no es una "cosita”, y en ese contexto puede responder más allá del papel de víctima desprovista de autonomía. Al manifestarse frente a la incomodidad, pasa a defenderse y a responderle al hombre, sin la mediación de una agresión física, pero en dicho contexto desagradable puede responder en el momento y ejercer su voz en el marco de una defensa pública. Esto me genera un poco de inquietud, pues parece que se está transmitiendo permanentemente la idea de una mujer víctima en aquellos relatos en los que se presentan a sí mismas como sujetos pasivos.

Lo que quisiéramos es un feminismo que esté más en competencia. Las mujeres no son seres débiles: no se trata de decir que cuando alguien se quiere acostar con una mujer necesariamente se trata una agresión, pues las mujeres deben y tienen la capacidad de defensa, más allá de sencillamente posicionarse desde el relato de la 
víctima. Es allí donde a mi juicio el movimiento $M e$ Too ha ido quizás en otra dirección. Cuando leemos algunos mensajes con el hashtag que se usa, en algunos casos uno dice: ¡caramba!, estamos hablando como si estuviera llegando el Tercer Reich y no creo que se trate de la misma dimensión. En algunos casos los hombres presentaron excusas y es así como expresan la petición del perdón, que en todo caso las mujeres no tienen por qué aceptar. Mi reflexión no quiere cerrar el tema, sino invitar a pensarlo. Hay que pensar en ello desde otras dimensiones.

E. SALAZAR: En la misma lógica del espectáculo y de la ligereza, de las redes sociales, etc., me llama la atención el paso de Donald Trump de la televisión a la política.

G. LIPOVETKSY: Él forma parte y participa en esa política del espectáculo, pero eso no explica nada, porque todas y todos lo hacen. De tal forma que el concepto de política-espectáculo sí la introduce él, pero Hillary Clinton también hizo espectáculo. En su caso estaban los grandes de Hollywood apoyándola. Recordemos las reuniones grandísimas que hacía con música, etcétera. La política espectáculo está en todas partes.

A pesar de todo, Trump ha propuesto una política del espectáculo de un tipo nuevo. Generalmente el espectáculo es una diversión agradable, simpática, pero lo que hizo Trump fue jugar al "chico malo". Entonces su espectáculo son agresiones, insultos, vulgaridades, y me atrevería a decir que de hecho se inventó algo. Rompió ese código washingtoniano en el que se usaba un lenguaje correcto o un lenguaje educado, prácticamente acusando a los mexicanos de violadores, a Hillary Clinton hay que llevarla a la cárcel, es decir, una vulgaridad increíble a la que reduce la política. Pero precisamente esa es su forma táctica, y esas agresiones le resultaron provechosas.

Ese juego es importante, pues le ha dado una imagen de credibilidad porque la gente quiere saber qué piensa Trump; en cambio, lo que dice Hillary Clinton parece con lógica, y en todo caso la imagen de ella no era buena. Pero sobre todo creo que el éxito de Trump, como el de Bolsonaro en Brasil, lo remite a fenómenos mucho más profundos de lo que sería la política espectáculo. Esto muestra, en primer lugar, que hay una nueva separación entre los que aceptan y se adaptan bien al fenómeno de vulgarización de la política, y los que no, y que además le temen a que se aproveche esta forma.

Quienes trabajan con símbolos, es decir los creativos, los profesores, los diseñadores, los empresarios, la gente que trabaja en la banca, no votaron por Trump. Ellos no tienen preocupaciones o inquietudes frente a sus condiciones generales de existencia, pues quizás estas son más estables. Pero hay un buen segmento de la 
población que en cambio no vive las cosas de la misma manera. La mundialización hizo que las industrias tradicionales tuvieran grandes problemas (Pittsburg o Chicago prácticamente se quedaron acabadas por la producción de los chinos), y gran parte de estas personas le temen al porvenir de su vida profesional. Vemos que los salarios suben de manera desperdigada, pero para quien se encuentra arriba de la pirámide y tiene unos salarios grandes, mientras que para los que se encuentran abajo no solo se estancan, sino que bajan. Hay dudas e inquietudes sobre lo que va a suceder en el futuro, así como hay preocupaciones en ese sentido por los hijos. Eso es algo nuevo.

En Europa están esos mismos factores de los que hablo, pero además con una inseguridad identitaria: fijémonos por ejemplo en el fenómeno de las migraciones, que le sucede a Trump también con los mexicanos. El último segmento de seguridad que es muy específico de Brasil es la enorme tasa de violencia; una violencia que genera prácticamente inseguridad diaria, cotidiana. Ahora que llego de São Paulo conversaba con la gente que me comentaba que a partir de ciertas horas no iban a determinados barrios porque hacerlo resultaría peligroso.

La victoria del Trump y del populismo se debe prácticamente a que él legitimó las preocupaciones de esa población, un segmento poblacional muy frágil que está preocupado y que piden que los escuchen, que piden respeto y protección. Considero que allí hay un resorte profundo. Todas esas causas van a perdurar, y es probable que en ese sentido aquel tipo de populismo esté todavía lejos de su desaparición.

E. SALAZAR: ¿Cómo leer la ligereza desde las diferencias entre las personas ricas y las personas empobrecidas, en las divisiones, proximidades y distancias en clave de clases sociales y modos de vida? Es evidente que cada persona o familia vive la posibilidad de la ligereza de maneras diferentes, pues hay situaciones socioeconómicas apremiantes.

G. LIPOVETKSY: Sí, claro que la viven de maneras diferentes. Digamos una cosa, en este caso, pienso yo a este nivel, habría dos fenómenos que se entrecruzan. Uno es que la cultura de la ligereza es un fenómeno que se ha propagado dentro de toda la sociedad; incluso las personas en pobreza económica la conocen en la televisión, en la publicidad, en los teléfonos inteligentes, las marcas, las modas. Las saben, las practican, pues incluso en los lugares más empobrecidos (como las favelas) se sabe lo que es. Ellos también quieren viajar, disfrutar de ciertos ideales de estilo de vida.

Por otro lado, está la pregunta necesaria frente al cómo hacerlo si no tienen los medios económicos. La diferencia radica en que las personas de las clases más pobres no podríamos venir, por ejemplo, a estos lugares [en la entrevista estamos 
en un Hotel Hilton en Bogotá], aunque si nos invitan, pues la cosa ya es diferente. Pero las personas pueden emplear sus propias maneras de acceder a esos modos de la ligereza y el placer, y buscar, por ejemplo, un AirBnB que se ajuste a sus presupuestos y deseos; van haciendo cuentas, cálculos, búsquedas detalladas, entre otras estrategias, que les permiten cierto nivel de acceso y gozo.

Sin embargo, la pregunta por la ligereza (la felicidad, el placer, el consumo) ha creado mucha ansiedad entre las personas y los consumidores. Es una suerte de ansiógeno. Hay mucha gente que no logra vivir de manera tranquila esta situación, pues se enfrenta a una tensión entre las urgencias económicas y los deseos y aspiraciones. Es decir, gente que se ve asfixiada porque tiene que pagar el arriendo de la vivienda, el transporte, los gastos de un carro, y el presupuesto con el que podría aguardar la posibilidad de ir a un restaurante se diluye. Muchas personas llegan a la tercera semana del mes y ya no tienen dinero que gastar, pues tienen empleos precarios o que no son suficientes para cubrir las responsabilidades de cada tipo de hogar. Las necesidades a partir del consumo se han desarrollado ampliamente, y para estas personas la ligereza se consume más a través de las imágenes que de su propia existencia material.

Evidentemente, este es el sentido de dicha paradoja de la realidad. Toda la civilización se cierne sobre la conservación de la liviandad, es decir, le dan paso a esta nueva manera de estar. Ya no se trata vivir exclusivamente en función las tareas pesadas. Por ejemplo, antes eran las mujeres quienes tenían que ir a lavar manualmente la ropa o desarrollar otras actividades pesadas del cuidado del hogar; ahora todo se puede hacer de manera más ligera, más liviana, y cada vez más personas pueden acceder a estas descargas. Sin embargo, y al mismo tiempo, para las familias conseguir esta ligereza, por ejemplo, de tener una lavadora, no se trata para nada de un asunto ligero. Al contrario, estas nuevas necesidades de búsqueda de la comodidad pueden operar como agentes ansiógenos.

Lo que menciono es algo que llama la atención, porque cuando uno lee los textos de los años cincuenta cuando la sociedad de consumo estaba comenzando, podemos evidenciar cómo los medios hablaban ampliamente de la ligereza, y sus promesas parecían casi milagrosas. A la gente le fascinaban los temas mediáticos frente a los vehículos, la televisión, los electrodomésticos, es decir, todo este mundo en apariencia más fácil y liviano. Pero en cambio hoy el tono dominante ya no es el de la modernidad y la promesa de facilidad, movimiento, libertad, pues el tinte fundamental lo pone la ansiedad. Está la ansiedad en relación con el dinero, y es por ejemplo evidente en las personas con empleos no muy rentables cuyos salarios están estancados 
o también en trabajadores precarizados, que pueden ver la ligereza pero no pueden aprovecharla.

Y por otro lado, la ligereza choca con otro código, ese sí no tan ligero. Se trata de aquel que tiene que ver con las normas de salud, que en muchos contextos son indignantes. Actualmente las personas están más informadas sobre qué comen, qué consumen, qué tipo de aire están respirando, y tener dicha información genera mayor ansiedad. Así, la ligereza implica una configuración paradójica entre la felicidad y la ansiedad, el deseo de tener al verlo tan cercano y la imposibilidad por la barrera de la precariedad. Esto fue justamente lo que quise mostrar en el libro.

E. SAlAzAR: En el problema de la ligereza, ¿qué papel ocupa el individuo, el sujeto, en la resolución de ese conflicto?

G. LIPOVETKSY: Considero que hay que establecer una distinción entre "la" ligereza y la ligereza. Fue Paul Valery quien dijo lo siguiente: hay que ser ligeros como el pájaro, no como la pluma. La ligereza del pájaro está dominada, mientras que la pluma se mueve sin más, va para cualquier lado porque depende únicamente de los vientos. Podríamos tomar esta metáfora como punto de partida.

Evidentemente, la civilización de la ligereza ha traído múltiples beneficios a la vida de las personas. Pero estamos en el momento en que debemos mostrar hasta dónde debe llegar, es decir, en dónde debe parar la promoción de la vida ligera. Uno no puede contentarse solo con la liviandad. La civilización debe reposar en otras normas diferentes: el trabajo, la inteligencia, la verdad, el saber. Eso no es la ligereza. Así que, primero, debemos rehabilitar esas normas y prácticas particularmente desde los sistemas educativos, y no sencillamente darle prioridad a la ligereza. Segundo, para los individuos propiamente es fundamental que las personas individualmente sienten que una existencia solo consumista no es satisfactoria, que no se compadece con el momento histórico que estamos viviendo. Se trata de profundizar las relaciones que tenemos con las cosas, con el tiempo, con la vida misma. Pensemos, por ejemplo, en las personas que se han volcado a la meditación de manera responsable, o a aquellas que están haciendo alpinismo como una forma de conexión consigo mismos. Necesitan construir a partir de estas búsquedas profundas, pues estas experiencias cuando son significativas se alejan radicalmente de la ligereza. Al practicarlas de este modo, se evidencia un camino, el proceso para completar algo.

Pero también creo que estamos en un momento en que no se trata de diabolizar la civilización de la ligereza, pero sí hay que inventar sistemas de ligereza que estén más controlados. Hay un ejemplo de muy fácil comprensión: si a alguien le gusta la música puede quizás aprender a tocar piano; oír pianistas es algo bello y ligero. Pero 
para lograr esa ligereza de tocar o de disfrutar el piano, habrá necesidad de trabajar antes. Lo que nos parece tan ligero necesita antes años de cultivo. Esto que menciono es muy del estilo Nietzsche, pues reconoce el proceso de construcción de un tipo de ligereza dominada, cultivada y controlada.

Créame: yo no soy ingenuo. No significa que aquella sea la felicidad. No caería en ese caso. Pero practicar la vida de ese modo también da momentos de felicidad, que es lo mismo. Esto es lo que les podemos proponer a las personas. Podemos brindarles los medios para que tengan momentos de felicidad, pues cuando uno está feliz se siente ligero. Pero hay que recordar que son momentos específicos, pues la vida no puede constituirse en su totalidad de dicho material. La felicidad absoluta no existe.

\section{Sobre el entrevistador}

Edward Salazar Celis. Sociólogo de la Universidad Nacional de Colombia. Magíster en Estudios Culturales de la Universidad de los Andes. Docente investigador de la Facultad de Ciencias Sociales de la Universidad Santo Tomás, Sede Principal, Colombia. Ganador del Premio al Mejor Trabajo de Grado en Sociología (2009), de la Beca Jóvenes Investigadores de Colciencias (2011-2012), de la Beca de Investigación en Fotografía del Ministerio de Cultura (2016), y segundo lugar en el concurso de novela Idartes-Emecé (2017). Investigador en estudios de moda, estéticas modernas, cultura visual, estilos de vida y clases sociales. Sus publicaciones más recientes son el catálogo curatorial “Acoger y amoblar: Estéticas e ideas sobre el hogar”, el documento "Estéticas en plural. La moda popular en Bogotá”, y el podcast “Nación Moda”, financiado por la Universidad Santo Tomás, Colombia.

\section{Referencias}

Alonso, L. y Fernández, C. (2010). Consumo e hipermodernidad: una revisión de la teoría de Gilles Lipovetsky. Anuario Filosófico, 43(2), 325-351.

Corral, R. (2007). Gilles Lipovetsky: una sociología del presente pos(hiper)moderno. Revista Casa del Tiempo, 1(1), 41-46.

Lipovetsky, G. (1986). La era del vacío: ensayos sobre el individualismo contemporáneo. Barcelona: Anagrama.

Lipovetsky, G. (1990). El imperio de lo efímero: la moda y su destino en las sociedades modernas. Barcelona: Anagrama.

Lipovetsky, G. (1998). La tercera mujer: permanencias y revolución de lo femenino. Barcelona: Anagrama. 
Lipovetsky, G. (2016). De la ligereza. Barcelona: Anagrama.

Posada, L. (2019). ¿Quién hay en el espejo? Lo femenino en la filosofía contemporánea. Madrid: Cátedra.

Sanín, C. (7 de febrero de 2018). La hija. Vice. Recuperado de https://www.vice.com/es_co/ article/j5b8nb/hija-columna-yo-tambien-me-too-carolina-sanin 